\title{
Prevalência de marcadores de trombofilia em pacientes portadores da síndrome de May-Thurner e trombose de veia ilíaca comum esquerda
}

\section{Prevalence of thrombophilia factors in patients with May-Thurner syndrome and left common iliac vein thrombosis}

Marcos Arêas Marques', Paulo Roberto Mattos da Silveira², Arno von Ristow 3 , Marcus Gress', Bernardo Massière ${ }^{4}$, Alberto Vescovi ${ }^{4}$, José Mussa Cury Filho ${ }^{4}$, Rafael Dias Vieira ${ }^{5}$

\section{Resumo}

Contexto: A relação entre trombose venosa profunda e trombofilia tem sido pouco estudada em indivíduos portadores de compressão da veia ilíaca comum esquerda, conhecida clinicamente como síndrome de May-Thurner.

Objetivo: Avaliar a prevalência de marcadores de trombofilia nos pacientes portadores de síndrome de May-Thurner e trombose de veia ilíaca comum esquerda.

Métodos: Entre março de 1999 e dezembro de 2008, 20 pacientes com síndrome de May-Thurner e trombose de veia ilíaca comum esquerda foram avaliados retrospectivamente quanto à presença de marcadores de trombofilia.

Resultados: Foi detectada a associação entre síndrome de May-Thurner e marcadores de trombofilia em 8 pacientes (40\%).

Conclusão: A presença de marcadores de trombofilia em pacientes com trombose de veia ilíaca comum esquerda e síndrome de May-Thurner é frequente, porém não difere da prevalência encontrada em pacientes portadores de trombose venosa profunda sem a síndrome associada.

Palavras-chave: Trombofilia; trombose venosa; fatores de risco.

\begin{abstract}
Background: The relationship between deep venous thrombosis and thrombophilia has been little studied in patients with left common iliac vein compression, clinically known as May-Thurner syndrome.

Objective: To evaluate the prevalence of thrombophilia markers in patients with May-Thurner syndrome and left common iliac vein thrombosis. Methods: From March 1999 to December 2008, 20 patients with May-Thurner syndrome and left common iliac vein thrombosis were retrospectively investigated for the presence of thrombophilia markers.

Results: The association between May-Thurner syndrome and thrombophilia markers was found in 8 patients (40\%).

Conclusion: There is a high prevalence of thrombophilia markers in patients with May-Thurner syndrome and left common iliac vein thrombosis. The prevalence, however, is not different from that found in patients with deep venous thrombosis without May-Thurner syndrome.
\end{abstract}

Keywords: Thrombophilia; venous thrombosis; risk factors.

Trabalho realizado no Ambulatório de Angiologia do Centro Integrado de Prevenção, Diagnóstico e Tratamento Vascular (CENTERVASC), Rio de Janeiro (RJ), Brasil.

${ }^{1}$ Chefe do Departamento de Angiologia do Centro Integrado de Prevenção, Diagnóstico e Tratamento Vascular (CENTERVASC); Membro do Conselho Científico da Sociedade Brasileira de

Angiologia e de Cirurgia Vascular (SBACV); Professor Associado do curso de Pós-Graduação em Cirurgia Vascular e Endovascular da Pontifícia Universidade Católica do Rio de Janeiro (PUC-

RJ), Rio de Janeiro (RJ), Brasil.

2Doutor em Medicina pela Escola Paulista de Medicina da Universidade Federal de São Paulo (UNIFESP), São Paulo (SP), Brasil; Professor associado do curso de Pós-Graduação em Cirurgia Vascular e Endovascular da Pontifícia Universidade Católica do Rio de Janeiro (PUC-RJ), Rio de Janeiro (RJ), Brasil.

${ }^{3}$ Diretor Médico do Centro Integrado de Prevenção, Diagnóstico e Tratamento Vascular (CENTERVASC); Professor Associado do curso de Pós-Graduação em Cirurgia Vascular e Endovascular da Pontifícia Universidade Católica do Rio de Janeiro (PUC-RJ), Rio de Janeiro (RJ), Brasil.

${ }^{4}$ Cirurgião Vascular do Centro Integrado de Prevenção, Diagnóstico e Tratamento Vascular (CENTERVASC); Professor Associado do curso de Pós-Graduação em Cirurgia Vascular e Endovascular da Pontifícia Universidade Católica do Rio de Janeiro (PUC-RJ), Rio de Janeiro (RJ), Brasil.

5Pós-Graduando em Cirurgia Vascular e Endovascular da Pontifícia Universidade Católica do Rio de Janeiro (PUC-RJ), Rio de Janeiro (RJ), Brasil.

Não foram declarados conflitos de interesse associados à publicação deste artigo.

Submetido em: 27.03.10, aceito em: 08.12.10

J Vasc Bras. 2010;9(4):229-232. 


\section{Introdução}

A síndrome da compressão da veia ilíaca (SCVI) descrita anatomicamente por May e Thurner em $1956^{1} \mathrm{em}$ estudo com cadáveres e clinicamente por Cockett em $1965^{2}$ - caracteriza-se pela compressão extrínseca da veia ilíaca comum esquerda pela artéria ilíaca comum direita contra o promontório (borda anterior da quinta vértebra da coluna lombar), podendo levar à formação de septos membranosos que determinam obstrução ao fluxo venoso do membro inferior esquerdo. Como consequência, pode haver o desenvolvimento de sintomas de desordem venosa crônica (DVC) secundária ou não à trombose venosa ilíaco-femoral $(\mathrm{TVIF})^{3}$. A prevalência dessa característica anatômica na população varia entre 22 e $33 \%$, segundo os dados fornecidos por May e Thurner na metade do século $20^{1}$. Kibbe observou uma prevalência de aproximadamente $25 \%$ de compressão maior que $50 \%$ da veia ilíaca comum esquerda de pacientes submetidos à tomografia computadorizada de abdome de emergência para avaliação de dor abdominal ${ }^{4}$. No entanto, a prevalência de manifestações clínicas ainda não foi estabelecida; acredita-se que esteja em torno de 2 a $3 \%$ da população ${ }^{5}$. Dois terços dos pacientes acometidos são mulheres, em sua maioria entre a segunda e quarta décadas de vida ${ }^{6}$.

Segundo Comerota, a TVIF está associada ao desenvolvimento de DVC severa, com sequelas físicas e sociais que levam a um impacto importante na capacidade produtiva desses pacientes ${ }^{7}$. Além disso, a TVIF está associada a um risco 2,6 vezes maior de recorrência se tratada de forma convencional com heparina, quando comparada a trombose venosa profunda (TVP) infrainguinal ${ }^{8}$. Portanto, o tratamento atual da TVIF consiste na resolução definitiva da obstrução mecânica ao fluxo venoso, mediante a remoção completa do trombo, visando diminuir o desenvolvimento dos sintomas da DVC, por meio da preservação da função das válvulas do segmento fêmoro-poplíteo, e prevenir a recorrência da trombose, podendo esses dois objetivos ser alcançados pela trombólise intratrombo direcionada por catéter ou por trombectomia direta com confecção de fístula arteriovenosa, seguida de angioplastia venosa com implante de stents autoexpansíveis ${ }^{7,9-11}$.

As trombofilias, por sua vez, são um conjunto de alterações hereditárias ou adquiridas do sistema hemostático que resultam em risco aumentado de eventos tromboembólicos, normalmente na presença de um fator desencadeante $^{12}$. A suspeita usualmente recai sobre os indivíduos que não possuem um fator de risco conhecido para eventos tromboembólicos (arteriais ou venosos) ou que apresentem
TVP em localizações que não nos membros inferiores. No acompanhamento de pacientes após episódio de TVP, é adequada uma avaliação criteriosa quanto à necessidade ou não de investigação de marcadores trombofílicos ${ }^{13}$. Essa avaliação é imprescindível, uma vez que a pesquisa sistemática e ampla sem seguir as recomendações dos principais consensos é onerosa para os serviços de saúde públicos ou privados. Além disso, um resultado falso positivo pode gerar exagerada e desnecessária ansiedade, assim como um falso negativo pode conduzir a uma equivocada sensação de segurança, deixando os pacientes ainda mais vulneráveis a um novo evento trombótico.

Na SCVI, o componente obstrutivo mecânico pode ser a única causa para a TVP e, nessa condição, o tratamento cirúrgico ou a trombólise seguida por um período determinado de anticoagulação seria suficiente para a resolução definitiva. No entanto, a alteração anatômica pode ser apenas o fator permissivo ${ }^{14}$ para que um marcador de trombofilia não diagnosticada se manifeste previamente; então, nesse caso, seria necessário um período de anticoagulação mais prolongado ou até mesmo perene, na dependência do marcador identificado.

O objetivo deste trabalho foi avaliar a prevalência de marcadores de trombofilia nos pacientes portadores de síndrome de May-Thurner e trombose de veia ilíaca comum esquerda.

\section{Métodos}

O estudo envolveu o período compreendido entre março de 1997 a dezembro de 2008, quando foram avaliados retrospectivamente 20 pacientes portadores de SCVI que desenvolveram trombose de veia ilíaca comum em membro inferior esquerdo e foram tratados pela equipe do Centro Integrado de Prevenção, Diagnóstico e Tratamento Vascular (CENTERVASC), sendo 16 mulheres e 4 homens, com idade média de 34,4 anos (14-51 anos). Todos os pacientes foram inicialmente rastreados para investigação da SCVI com eco Doppler colorido e, posteriormente, confirmados com angiorressonância magnética, angiotomografia computadorizada ou flebografia.

A investigação de marcadores de trombofilia nesses pacientes incluiu a pesquisa do fator $\mathrm{V}$ de Leiden (homozigoto ou heterozigoto) e da mutação 20210 da protrombina (homozigota ou heterozigota), deficiências das proteínas C e $\mathrm{S}$ e antitrombina, hiper-homocisteinemia e a pesquisa dos anticorpos anticardiolipina (IgM e IgG) e anticoagulante lúpico. Não foi feita a pesquisa da anti- $\beta 2$ glicoproteína I (IgM e IgG). 


\section{Resultados}

Dos 20 pacientes com diagnóstico confirmado da SCVI associada à trombose de veia ilíaca comum esquerda, $16(80 \%)$ eram do sexo feminino e quatro $(20 \%)$ eram do sexo masculino. A pesquisa de marcadores de trombofilia foi positiva em 8 desses pacientes (40\%), sendo 7 $(87,5 \%)$ do sexo feminino e $1(12,5 \%)$ do sexo masculino. A incidência dos marcadores de trombofilia nos 8 casos está expressa na Tabela 1, sendo diagnosticada de forma isolada em 7 pacientes $(87,5 \%)$ e de forma associada em 1 paciente (12,5\%). Em relação ao tipo de marcador, houve predomínio dos anticorpos antifosfolipídios (anticardiolipina e anticoagulante lúpico), sendo estes identificados em $5(62,5 \%)$ pacientes. O Consenso de Sidney foi utilizado como referência ${ }^{15}$.

\section{Discussão}

A etiologia da TVP deve ser sempre considerada multifatorial, resultante de uma interação entre as características genéticas do paciente e de fatores de risco que serviriam de gatilho para a sua manifestação. No caso dos pacientes portadores de SCVI com trombose de veia ilíaca comum esquerda, discute-se a possibilidade de a SCVI servir de gatilho para a manifestação clínica dos marcadores de trombofilia ou de ela ser o agente etiológico único, como sugeriu Verhaeghe ${ }^{16}$. Há de se ressaltar o fato de haver uma dissociação entre a frequência da presença da SCVI em estudo com cadáveres ${ }^{1,17}$ e o desenvolvimento dos sintomas ${ }^{14,18}$.

Neste trabalho, podemos observar um predomínio de pacientes do sexo feminino (80\%), com média de idade de 34,4 anos. Esse achado está de acordo com a descrição clássica da SCVI que é relatada como uma condição mais prevalente no sexo feminino e na terceira ou quarta décadas de vida ${ }^{4,18-20}$.

A prevalência de marcadores de trombofilia neste trabalho também não difere da encontrada por autores que pesquisaram a incidência desses marcadores em pacientes com TVP de membros inferiores sem a síndrome associada $^{21,22}$.

\section{Conclusão}

Levando-se em consideração que a TVP é uma doença de etiologia multifatorial, devemos considerar que a SCVI serve como um fator permissivo para que a presença de um ou mais marcadores trombofílicos se manifeste
Tabela 1 - Incidência dos nove marcadores de trombofilia nos oito pacientes portadores de síndrome de May-Thurner e trombose venosa profunda ilíaca comum esquerda

\begin{tabular}{lc}
\hline Marcador de trombofilia & Número \\
\hline Anticoagulante lúpico & 3 \\
Anticardiolipina & 2 \\
Fator $\vee$ de Leiden heterozigoto & 2 \\
Mutação 20210 da protrombina heterozigoto & 2 \\
\hline
\end{tabular}

clinicamente e que a prevalência destes nos pacientes com trombose de veia ilíaca esquerda e SCVI é frequente, porém não difere da encontrada em pacientes portadores de TVP sem a síndrome associada.

\section{Referências}

1. May R, Thurner J. Ein Gefäßsporn in der vena iliaca comunis sinistra als ursache der überwiegend linksseitigen beckenven-thrombosen. Zeitschrift Für Kreislauf-Forschung. 1956;45:912-922.

2. Cockett FB, Thomas ML. The iliac compression syndrome. $\mathrm{Br}$ J Surg. 1965;52:816-21.

3. Kibbe MR, Ujiki M, Goodwin AL, Eskandari M, Yao J, Matsumura J. Iliac vein compression in an asymptomatic patient population. J Vasc Surg. 2004;39:937-43.

4. Sandri JL. Síndrome de May-Thurner - Tratamento Endovascular. In: Brito C). Cirurgia Vascular. Rio de Janeiro: Revinter; 2008. p. 1653-64.

5. Wolpert LM, Rahmani O, Stein B, Gallagher J), Drezner AD. Magnetic resonance venography in the diagnosis and management of MayThurner syndrome. Vasc Endovascular Surg. 2002;36:51-7.

6. Neglén P. Chronic deep venous obstruction: definition, prevalence, diagnosis, management. Phlebology. 2008;23:149-57.

7. Comerota AJ, Gravett MH. Iliofemoral venous thrombosis. J Vasc Surg. 2007; $46: 1065-76$

8. Douketis JD, Crowther MA, Foster GA, Ginsberg JS. Does the location of thrombosis determine the risk of disease recurrence in patients with proximal deep vein thrombosis? Am J Med. 2001;110:515-9.

9. Grunwald MR, Goldberg MJ, Hofmann LV. Endovascular management of May-Thurner syndrome. Am J Roentgenol. 2004;183:1523-4.

10. Bjarnason $H$. Endovascular treatment for chronic iliofemoral venous obstruction. Perspect Vasc Surg Endovasc Ther. 2004; 16:223-32.

11. França LHG, Tavares V. Insuficiência venosa crônica. Uma atualização. J Vasc Bras. 2003;2:318-28.

12. Heit JA. Thrombophilia: common questions on laboratory assessment and management. Hematology Am Soc Hematol Educ Program. 2007:127-35.

13. Paschôa AF. Impacto da pesquisa laboratorial de trombofilia na prevenção secundária e orientação dos doentes com tromboembolismo venoso. J Vasc Bras. 2006;5:331-2. 
14. Raju S, Neglen P. High prevalence of nonthrombotic iliac vein lesions in chronic venous disease: a permissive role in pathogenicity. J Vasc Surg. 2006;44:136-43.

15. Miyakis S, Lockshin MD, Atsumi T, et al. International consensus statement on an update of the classification criteria for definite antiphospholipid syndrome (APS). J Thromb Haemost. 2006;4:295-306.

16. Verhaeghe R. lliac vein compression as an anatomical cause of thrombophilia: Cockett's syndrome revisited. Thromb Haemost. 1995;74:1398-401.

17. May R, Thurner J. The cause of the predominantly sinistral occurrence of thrombosis of the pelvic veins. Angiology. 1957:8:419-27.

18. Knipp BS, Ferguson E, Williams DM, et al. Factors associated with outcome after interventional treatment of symptomatic iliac vein compression syndrome. J Vasc Surg. 2007;46:743-49.

19. Fazel R, Froehlich JB, Williams DM, Saint S, Nallamothu BK. Clinical problem-solving. A sinister development--a 35-year-old woman presented to the emergency department with a 2-day history of progressive swelling and pain in her left leg, without antecedent trauma. N Engl I Med. 2007;357:53-9.

20. Ludwig B, Han T, Amundson D. Postthrombotic syndrome complicating a case of May-Thurner syndrome despite endovascular therapy: case report and review. Chest. 2006;129:1382-6.
21. Auerbach AD, Sanders GD, Hambleton J. Cost-effectiveness of testing for hypercoagulability and effects on treatment strategies in patients with deep vein thrombosis. Am J Med. 2004;116:816-28.

22. Marques MA, da Silveira PRM, Ristow A, et al. Pesquisa de marcadores de trombofilia em eventos trombóticos arteriais e venosos: registro de 6 anos de investigação. J Vasc Bras. 2009;8:225-31.

Correspondência:
Marcos Arêas Marques
Rua Sorocaba, 464/308 - Botafogo,
CEP 22610-110 - Rio de Janeiro (RJ), Brasil
E-mail: mareas@centervasc.com.br
Contribuições dos autores
Concepção e desenho do estudo: MAM, PRMS
Análise e interpretação dos dados: MAM, PRMS
Coleta de dados: MAM, PRMS, RDV
Redação do artigo: MAM, PRMS, AR, MG, BM, JMCF, AV, RDV
Aprovação final do artigo*: MAM, PRMS, AR, MG, BM, JMCF, AV, RDV
Análise estatística: N/A
Responsabilidade geral pelo estudo: MAM
Informações sobre financiamento: N/A
*Todos os autores leram e aprovaram a versão final submetida ao J Vasc Bras

